\title{
USE OF AN ALIASING ARTEFACT IN FT SPECTROMETRY TO DISTINGUISH BETWEEN PUISED AND CONTINUOUS EMISSIONS OCCURING IN THE SAME SOURCE
}

\author{
R. STRINGAT, G. FABRE, J.M. MOUREAUX and A.J. ROSS* \\ Laboratoire d'Optique Atomique et Moléculaire, Faculté des Sciences, Parc Valrose, \\ F-06034 Nice cedex, France \\ *Laboratoire de Spectrométrie Ionique et Moléculaire, Université Lyon I, 43 boulevard \\ du 11 novembre 1918, F-69622 Villeurbanne cedex, France
}

\begin{abstract}
Spectral aliasing can be usefully employed to differentiate between emission from pulsed and continuous sources when recording spectra on a continuous scanning Fourier transform spectrometer. Examples are given using a pulsed copper vapour laser to excite fluorescence in the presence of strong blackbody radiation.

The continuous emission is sampled at regular optical path differences $\Delta \delta$ and shows no aliasing in the spectral range $0-\frac{1}{2 \Delta \delta}$
\end{abstract}

The pulsed emission is slaved to the interferometer at a frequency which is half that of the interferometer sampling rate producing a duplicate of the pulsed spectrum $B(\sigma)$ at $B\left(\frac{1}{2 \Delta \delta}-\sigma\right)$.

The two can thus be distinguished, provided that the ghosts appear in a spectral region which contains no true lines.

It is well known that, in symmetric interferometry, the spectral intensity $\mathrm{B}(\sigma)$ of the light source (where $\sigma$ is given in wavenumbers) is the cosine Fourier transform of the interferogram:

$$
\mathrm{B}(\sigma)=\int_{0}^{\infty}\left[\mathrm{I}(\delta)-\frac{1}{2} \mathrm{I}(0)\right] \cos (2 \pi \sigma \delta) \cdot \mathrm{d} \delta
$$

where $I(\delta)$ represents the intensity of light detected after interference, as a function of path difference $\delta$ (BELL R.J. "Introductory Fourier transform spectroscopy", Academic Press, 1972). 
If the interferogram is sampled at regular intervals $\Delta \delta(\delta=k \Delta \delta$, with $k$ integer), the Fourier transform is a periodic function, with a period $\frac{1}{\Delta \delta}$ :

$$
\cos (2 \pi \sigma k \Delta \delta)=\cos \left[2 \pi\left(\sigma+\frac{\mathrm{m}}{\Delta \delta}\right) \mathrm{k} \Delta \delta\right]
$$

where $\mathrm{m}$ takes integral values.

Because $B(\sigma)$ is an even function, we have $B(\sigma)=B\left(\sigma^{\prime}\right)$ if $\sigma^{\prime}=\frac{1}{\Delta \delta}-\sigma$

$$
\cos \left(2 \pi \sigma^{\prime} \mathrm{k} \Delta \delta\right)=\cos \left[2 \pi\left(\frac{1}{\Delta \delta}-\sigma\right) \mathrm{k} \Delta \delta\right]=\cos [2 \pi(-\sigma) \mathrm{k} \Delta \delta]=\cos (2 \pi \sigma \mathrm{k} \Delta \delta)
$$

The consequence is that in the range $0-\frac{1}{\Delta \delta}$, the spectrum exhibits reflection symmetry with respect to the median, $\frac{1}{2 \Delta \delta}$.

To avoid any ambiguity in the assignment of spectral lines, the FT calculation is usually mathematically limited to an interval $0-\frac{\sigma_{M}}{2}$ (where $\sigma_{M}=\frac{1}{\Delta \delta}$ ) which contains the spectrum to be studied.

Then, if the spectrum of a pulsed source slaved to the spectrometer (STRINGAT R., FABRE G., CIRIO L. and ROSS A. Optics Comm. 1991, 81, 179) is recorded when the repetition rate of the source is half the sampling rate of the interferometer, the period of the Fourier transform function for this source becomes $\frac{1}{2 \Delta \delta}=\frac{\sigma_{M}}{2}=\sigma_{M}^{\prime}$ and aliasing occurs, i.e. an artefact spectrum is produced at $\sigma^{\prime}=\frac{1}{2 \Delta \delta}-\sigma=\sigma_{M}^{\prime}-\sigma$, symmetrical with respect to the true spectrum by reflection in $\frac{\sigma_{M}^{\prime}}{2}=\frac{\sigma_{M}}{4}$.

In the presence of both a continuous source, which will of course be sampled at chosen intervals $\Delta \delta$ of the interferometer, and a pulsed source which is sampled at intervals $2 \Delta \delta$, aliasing will occur only for the pulsed spectrum. This experimental arrangement can be put to use in separating the spectrum associated with the pulsed source from that of the continuous source. The combination of pulsed and continuous sources is common, and one of them -usually the continuous one- is often undesirable 
(blackbody radiation, chemiluminescence, electrical discharge...). This may be advantageous if the continuous component is likely to vary in composition with time; for example, the substraction of a subsequent "blackbody contribution" spectrum can produce a poor baseline if the temperature varied during the experiment. Of course, the spectral region to be studied will require a careful choice of detectors and optical filters to ensure that the true spectrum (continuous and pulsed) lies entirely to one side of $\frac{\sigma_{M}}{4}$ so that there is no overlap between the spectrum and the alias of the pulsed emission, and hence no ambiguity possible in assignment.

The position of the moving mirror in the spectrometer we used (BOMEM DA3) is monitored by the fringes of a $\mathrm{He}-\mathrm{Ne}$ reference laser, $\lambda_{\mathrm{R}}$, detected on an auxiliary detector. The possible sampling intervals $\Delta \delta$ are $\lambda_{R}, \frac{\lambda_{R}}{2}, \frac{\lambda_{R}}{4}$ and $\frac{\lambda_{R}}{8}$, and the corresponding spectral ranges, $0-\frac{\sigma_{M}}{2}$, are respectively $0-7899,15798,31596$ and $63192 \mathrm{~cm}^{-1}$. Each sampling of the intefierogram gives rise to a TTL signal from the interferometer. The frequency of these TTL pulses is halved before triggering the pulsed source. Given that $\mathrm{I}(0)$ should appear in the data points of the interferogram for the pulsed source, it is essential to select always the even points on the interferogram $(k=0 \rightarrow \delta=0)$ during successive scans. The mirror always returns to an even number (multiple of 256) of He-Ne fringes before the zero path difference position when a scan has been completed, so the number of sampling pulses occuring before $\delta=0$ is necessarily even. Thus, with an electronic system which eliminates every other pulse but automatically retains the first (for which $\mathrm{k}$ is even), we are certain that the pulsed source will be triggered at $\delta=0$. A second signal from the interferometer at the end of each scan is used to reinitialize the frequency divider when a scan has been completed.

We give examples of spectra taken using a copper vapour laser (Oxford Lasers $\mathrm{Cu} 25$ ) having a repetition rate $6.32 \mathrm{kHz}$, and using a sampling rate of $12.64 \mathrm{kHz}$ in the interferometer to generate the signals necessary to trigger the pulsed source. It is clear that the $S / N$ ratio in the "true" lines and "artefact" lines is conserved in the procedure. 

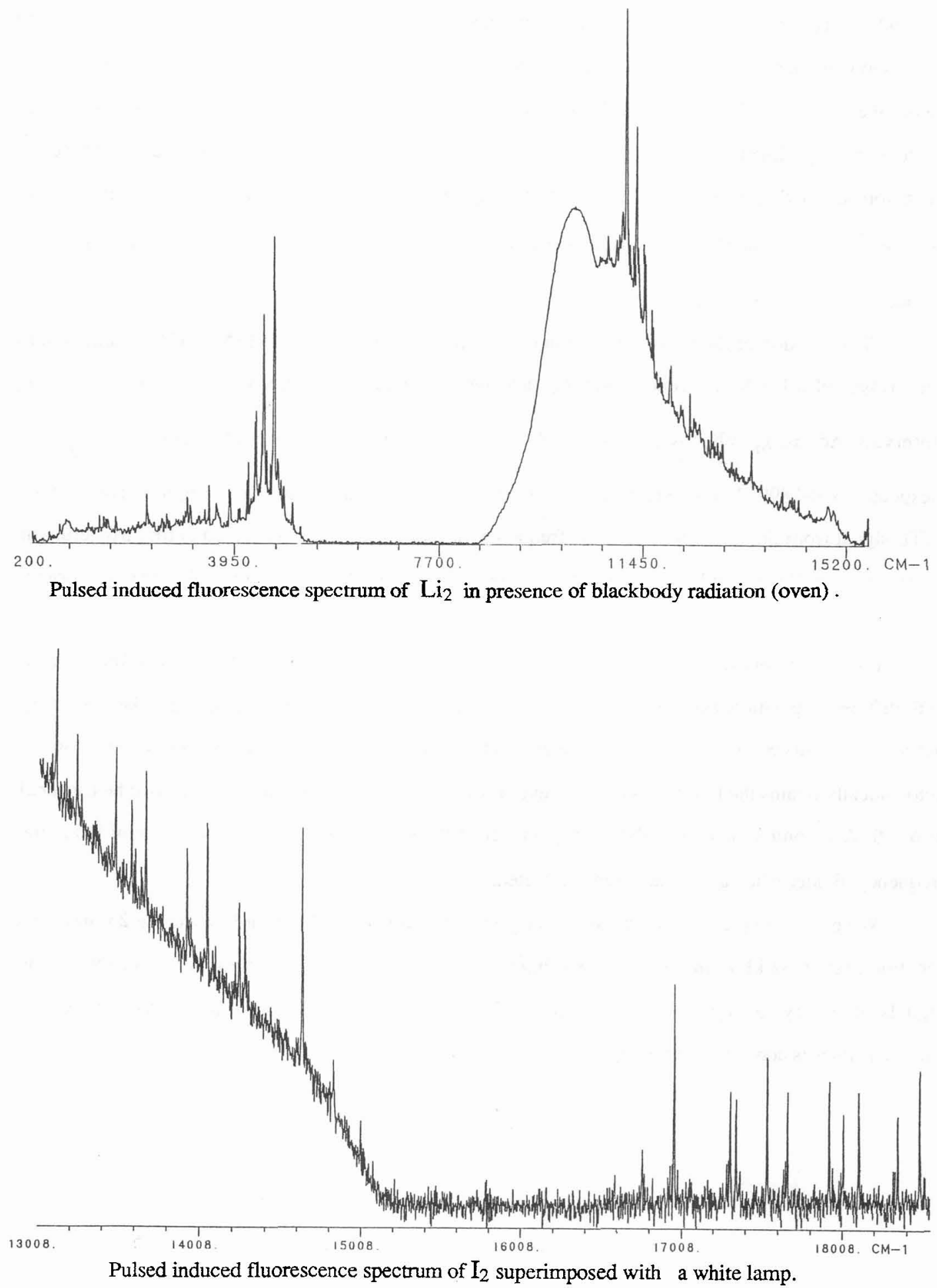